\title{
Independence of sensitivity on different foveal areas*
}

\author{
JAMES F. COLLINS \\ University of Missouri-Kansas City, Kansas Ciț, Missouri 64110
}

\begin{abstract}
Evidence suggests that for close retinal areas a correlation in sensitivity exists between the areas. Correlations as a function of distance from fixation and between areas were studied. In Experiment l, both forms were equidistant from fixation and five different distances apart. In Experiment II. both forms fell on an imaginary line through fixation. The forms usually did not fall on equally sensitive areas as in Experiment l. Both experiments showed that accuracy was lower for two- than for one-form displays. Closer forms had the lowest accuracy, suggesting perhaps mutual contour masking. However, in the sense of an intratrial correlation, no significant relationships were found. These two types of independence were discussed in terms of contour masking and varying sensitivity.
\end{abstract}

At any given point in time, all photoreceptors in the retina are not equally sensitive to light. Spontaneous receptor firings, different refractory periods, lateral inhibition, and varying stages of dark adaptation can account for considerable differences in thresholds for individual fibers. Consequently, a dim spot of light might have sufficient energy to evoke a response in one receptor but not in another at a given moment, even though the two receptors might be equally sensitive when averaged over time.

Those serving as Ss in tachistoscopic experiments realize that the same phenomenon exists for retinal areas considerably larger than one receptor. For example, assume that a $S$ is visually fixated on a small black " $X$ " in the center of a blank field. Four capital letters are briefly exposed, each appearing in a different quadrant of two perpendicular axes centered on, and equidistant from, the "X." In the fovea at least, groups of receptors spaced equidistant from center are approximately equally sensitive. Thus, all letters should appear equally clear; but this is seldom the case. Virtually always, some letters appear to be clearer than others.

Recent research has attributed this to varying sensitivity on different retinal areas at a given point in time. Thus, if the noise level reducing sensitivity in one area is high at the moment when a brief tachistoscopic presentation is made in that area, perception will be impaired. The simultaneous presentation of another letter in a highly sensitive area will lead to a clear perception.

The exact causes of this varying sensitivity are not of concern here. The aspect considered was whether it is random and independent in different areas at a given moment if the two areas are sufficiently separated spatially.

Much of the work on uncorrelated sensitivity in different areas came from the work of Collins and

*This research was supported by National Institutes of Health Grant MH-1206. This manuscript was submitted to the faculty of the Psychology Department at the University of Illinois in partial fulfillment of the requirements for the $\mathrm{PhD}$ degree.
Eriksen (1967), who studied the exposure duration required to identify correctly on $80 \%$ of the trials all forms in one-, two-, three-, and four-form displays. The forms appeared within the corners of the square and were $1 / 2$ deg of visual angle from a central fixation point. It was found that the two-, and three-, and four-form displays required the same exposure duration for correct identification of all forms in the displays on $80 \%$ of the trials. The exposure time necessary for one-form displays was about $14 \mathrm{msec}$, and the other three types of displays required about $18 \mathrm{msec}$. This difference was attributed partially to the higher probability of guessing correctly when only one form was presented, or perhaps to the ability of the $S s$ to consider their responses more carefully when only one was required.

In another part of the experiment, the display elements were farther from center within the corners of 3/4- and 1-deg squares. In these conditions, the equality of two-, three-, and four-form displays found in the $1 / 2-\mathrm{deg}$ condition disappeared. A consistent decrease in accuracy was now found as more forms were added. This result was consistent with the concept of uncorrelated sensitivity on areas of the retina separated by some minimal distance such as $3 / 4 \mathrm{deg}$. When the forms were close, as in the $1 / 2$-deg square, the sensitivity levels were highly correlated. Thus, if one form was perceived correctly, there was a high probability that the others would be also. Likewise, if noise interfered with one, it would probably interfere with the others. In the larger displays, the forms fell on areas with lower or zero sensitivity correlations. The probability that these areas would all simultaneously have high sensitivity would be relatively low, and thus the probability that all forms would be perceived clearly at one time would be lowered. An independent probability model fit the data for the I-deg displays quite closely, suggesting that at this separation the error components were essentially independent. Thus, if the probability of any given letter being masked by random noise were .2 , this probability would remain the same regardless of whether or not other forms were masked. The probability of four being identified would be $(.8)^{4}$, or approximately .41 . 
Eriksen (1966) found that two-form hit rates could be predicted quite accurately from one-form hit rates, assuming uncorrelated sensitivities. This was as true where the two forms were the same (i.e., two As) as it was where they were different (i.e., an $A$ and a $U$ ).

Eriksen and Lappin (1965) used up to six repetitions of the same form in a circular display. The obtained hit rates were predicted quite well by the independence model. Estes and Taylor (1966) also obtained evidence for independence using redundant target forms. As long ago as 1948, Schlosberg (1948) advanced the concept to account for a S's ability to detect one to four dots in a display.

Eriksen and Lappin (1967) studied displays in which the simultaneous forms were not necessarily redundant. The independence model was again found to fit the data. In both types, there is strong support for independence when the forms are sufficiently separated. However, Collins and Eriksen (1967) found evidence suggesting correlation in sensitivity when the forms were placed relatively close to fixation $(1 / 2 \mathrm{deg})$. This study gave approximate limits of correlations in sensitivity. However, no systematic study has been made of the size of these correlations as a function of (1) distance between forms and (2) distance of the forms from fixation.

The present study examined these two factors in two experiments. The first dealt with forms which fell on the circumference of an imaginary circle centered on fixation. Both were the same distance from fixation but varying distances apart. Experiment II studied relationships between stimuli falling on a lateral line through fixation, again at varying distances from each other and also from fixation.

\section{METHOD}

\section{Experiment I}

The Ss were four paid graduate students at the University of Illinois, with normal or corrected to normal vision. All had served previously in tachistoscopic experiments.

Two fields of a Scientific Prototype Model GA three-field tachistoscope were used for stimulus presentation. The forms used were the black capital letters A. H, O, T, and Y (Paratipe No. 11316) mounted on white translucent plastic cards. Each letter subtended $.2 \mathrm{deg}$ of visual angle. Five sets of single-form displays were constructed such that for each set. each of the five forms appeared once in each of six different positions on an imaginary circle whose center was on fixation. Thus, there were 30 cards in each of the five sets. Each set was made based on circles of different size whose radii were $1 / 4,1 / 2,3 / 4,1$, and $1 \frac{1 / 4}{4}$ deg of visual angle. Double-form stimuli were constructed not only as a function of circle size. but also according to the distance between the two forms: $1 / 4.1 / 2,3 / 2$. and $1 \mathrm{deg}$. For each of the four largest circles. 25 stimulus cards were constructed for each combination of circle size and distance between forms ( 16 sets of 25 cards eachl. The maximum possible separation between forns for the ${ }^{1}$-deg circle was $1 / 2 \mathrm{deg}$. so only two sets of 25 each were prepared: $1 \%$ and $1 / 2$ deg between forms. The identity of each form on a display was independent of the other so that a $S$ s: perception of one form gave no information about the identity of the other. lorm identity was also independent of location on the circle.
Each S served for a total of $28 \mathrm{t} / 2$-h sessions. For each trial in a session, the $S$ was instructed to fixate a small illuminated $X$ in the center of a dark field. After a ready signal from $E$. $S$ triggered the stimulus onset and responded appropriately. The illumination of the stimulus field and fixation $\mathrm{X}$ was $5 \mathrm{~mL}$. The $S$ always knew beforehand how many and on what size circle the forms would appear but not the location of the form or forms. For the two-form displays, he was also uninformed of the distance between forms.

The first two sessions were used for determining an exposure duration necessary for the $S$ to attain an accuracy level of approximately $80 \%$ on single-form stimuli on a particular size circle. During the next four sessions, data were collected on the double-form stimuli for that circle size. The four sets of 25 were randomized by shuffling, and presented in four blocks of 25 trials each. An additional block of the appropriate 30 single-form stimuli was presented making a total of five data blocks per session. After these first six sessions, this procedure was repeated on a different size circle until all had been presented. Because of the smaller number of double stimuli with the $1 / 4-$ deg radius, only two double-stimuli data sessions were run. The order of presentation of different size circle was counterbalanced over Ss to control for order effects. The position of the single-stimulus block in the series was counterbalanced over sessions and Ss.

\section{Experiment II}

Since previous work had dealt exclusively with correlations in sensitivity of forms equidistant from fixation, an attempt was made to determine the relationships of forms falling on an imaginary straight line through the fixation point. In this case, the two forms would not necessarily fall on equally sensitive foveal areas. Distance from fixation and distance between forms could not be completely balanced. For example, if the forms are $1 / 2$ and $3 / 4$ deg from fixation, respectively, the lateral distance between them could be $1 / 4$ deg but not $1 / 2$ deg. However, it was still possible to study correlations present at various locations, so all possible lateral combinations of $1 / 4$ and $1 / 2$ deg between forms were studied. In Experiment II, the exposure durations for the various distances from center were not different. Both forms appeared in the same field, so a single exposure time was necessarily used. Thus, some differential accuracy as a function of distance from center was expected. However, equal hit rates are not a necessary prerequisite for studying independence between forms.

The same four Ss each served for an additional 15 sessions. Initially, all 150 single-form stimuli from Experiment I 16 positions $\times 5$ forms $\times 5$ circle sizes) were shuffled randomly for concurrent presentation. During the first four sessions. an exposure duration was established such that the average accuracy rate for these 150 single-form displays from all five circle sizes was approximately $75 \%$. In the next two sessions, data were collected for the double-form displays. As in Experiment 1.25 stimulus cards were constructed for each combination of radial distances (i.e., $3 / 4$ and $1 \mathrm{deg}$ from fixation). which were either ${ }^{1}+$ or $1 / 2 \mathrm{deg}$ apart. The next session and every third thereafter. the 150 single-form stimuli were run again to check on the stability of the exposure duration established for each $\mathrm{S}$. Table 1 shows the spacings of the eight double-form conditions run in Experiment II. Four of these eight were presented in each double-form session. as determined by counterbalancing over sessions and Ss. The order of stimulus presentation within a block was randomized by shuffling the stimulus cards.

\section{RESULTS}

The exposure durations for $80 \%$ accuracy on one-form displays obtained in Experiment I were examined in a two-way analysis of variance (circle size and $\mathrm{Ss}$ ). The l'd-deg circle was slightly more difficult. 
Table 1

Distance from Fixation Point of Each Form for the Different Conditions of Experiment II

\begin{tabular}{cccccccc}
\hline Condition & 1 & 2 & 3 & 4 & 5 & 6 \\
\hline Distance from Fixation in First Form & $1 / 4$ & $1 / 2$ & $3 / 4$ & 1 & $1 / 4$ & $1 / 2$ & $3 / 4$ \\
Degrees of Visual Angle in Second Form & $1 / 2$ & $3 / 4$ & 1 & $1 \frac{1 / 4}{3 / 4}$ & $1 / 4$ \\
\hline
\end{tabular}

but the difference did not reach significance $[F(4,12)=$ $2.08, p>.05]$. The average durations across the four $\mathrm{Ss}$ were $3.4,3.1,3.0,3.3$, and $3.8 \mathrm{msec}$ for the $1 / 4$ - through 11/4-deg circles, respectively. The effect of Ss was significant $[\mathrm{F}(3,12)=42.25, \mathrm{p}<.01)$, indicating differences among the Ss in overall acuity. The mean exposure times of each of the four Ss over the five different circle sizes were $2.7,3.4,2.4$, and $4.7 \mathrm{msec}$. These exposure times are considerably less than those established in the Collins and Eriksen study due to a difference in stimulus field luminance $(5 \mathrm{~mL}$ here and only $.2 \mathrm{~mL}$ in the earlier study).

A comparison of single- and double-form accuracy was made in a three-way analysis of variance (single-double forms, circle size, and Ss). Accuracy decreased when two-form displays were presented (77\% for one form, $72 \%$ for two forms), but not significantly $[F(1,3)=4.71, p>.05]$. The effect of circle size was also nonsignificant $[\mathrm{F}(4,12)=1.57)$. This was anticipated, since sensitivity differences that may exist at various distances from fixation were compensated for by the different exposure durations. The effect of Ss was significant $[F(3,12)=4.1, p<.05]$, as was the Single-Double Forms by $S$ interaction $[F(3,12)=8.2$, $\mathrm{p}<.011$. The $\mathrm{S}$ main effect suggests that the $\mathrm{Ss}$ were not matched exactly for overall accuracy level. The interaction appears to be due mainly to one $S$, who dropped from $77 \%$ accuracy on one form to $66 \%$ for two forms, while the other three Ss dropped considerably less. Thus, there would appear to be individual differences in the ability of Ss to process two forms simultaneously as opposed to only one. The other two interactions did not reach significance.

In order to study the effects of distance between forms, the single-form data were dropped and the double-form data were analyzed in a four-way analysis of variance (circle size, distance between forms, first and second forms, and Ss). First-second referred to the order in which the $S$ was instructed to report the letters (clockwise). The main effect of circle size was not significant at the .05 level but did reach significance at the .10 level $[F(3,9)=3.57]$. Again, the evidence shows that the initial time settings for $80 \%$ accuracy fairly well matched the various foveal areas for visibility. The effect of distance between forms was significant $[F(3,9)=$ $9.81, \mathrm{p}<.01]$, with fewer errors being made where the distance was $3 / 4$ or $1 \mathrm{deg}$. This suggests that some form of mutual contour masking might have occurred when the forms were closer together. Figure 1 shows the accuracy levels obtained as a function of the five radius sizes and four distances between forms.
The effect of first or second form reported did not approach significance, demonstrating that there was no apparent tendency for response order to influence the Ss' perception of one of the pair at the expense of the other. The $S$ effect was again significant $[F(3,27)=$ $12.02, \mathrm{p}<.01]$, with mean accuracy levels ranging from $67 \%$ to $74 \%$. All second-order interactions were nonsignificant except for Circle Size by First-Second $[F(3,9)=4.77 ; p<.05]$ and Circle Size by Ss $[F(9.27)$ $=3.21, \mathrm{p}<.01]$. Upon closer examination, the second stimulus was found to be perceived slightly better with circle sizes of $1 / 2$ and $3 / 4 \mathrm{deg}$ and slightly worse at 1 and $1 \frac{1}{4} \mathrm{deg}$. No interpretation was made of these small differences, and the effect was attributed to chance factors. No meaningful trends were found in the Circle by $\mathrm{S}$ interaction. All of the third-order interaction Fs were less than 1 .

The primary analysis was to have been a measure of correlation between the two forms on a display as a function of circle size, distance between forms, and Ss. In this case, with two dichotomous measures (right-wrong on first and second reported forms) and where an underlying continuous distribution can reasonably be assumed, the tetrachoric correlation is the appropriate correlation coefficient (McNemar, 1963). Accordingly, the data from the 100 two-form display trials run under each of the 72 possible conditions were placed in 2 by 2 tables (order of report by right-wrong). Contrary to expectation, there were no indications of a significant correlation, either positive or negative, under

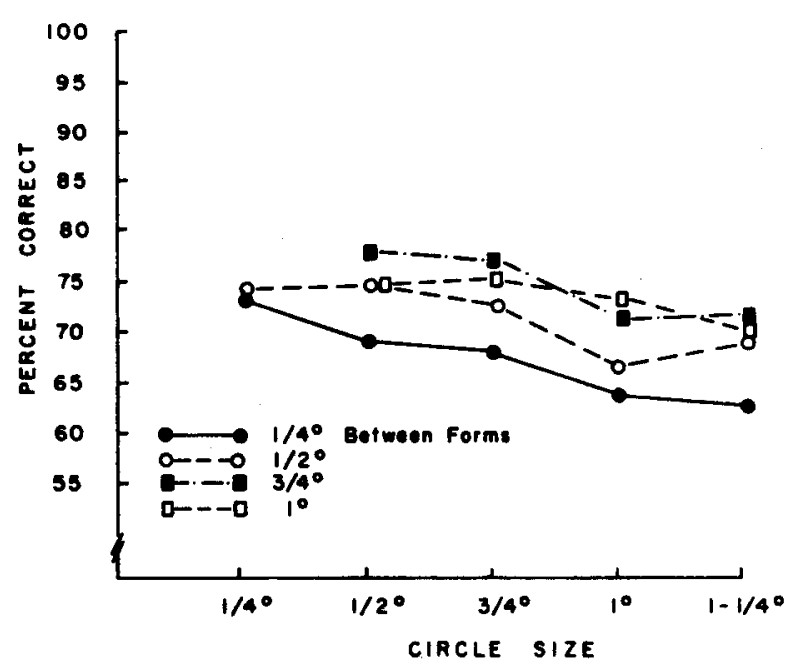

Fig. 1. Accuracy in Experiment I as a function of circle size and distance between forms. 


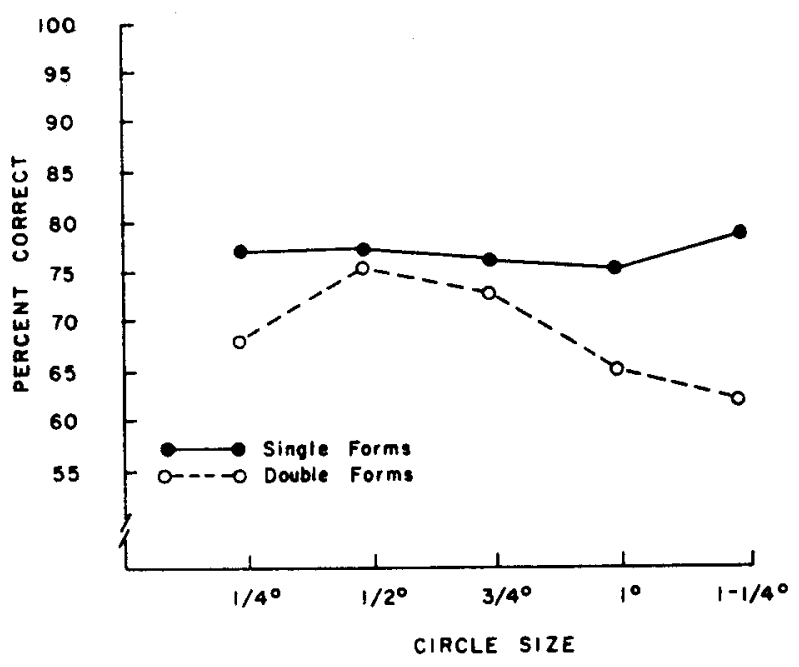

Fig. 2. Accuracy in Experiment II as a function of circle size and number of forms.

any conditions. Out of the 72 tables, the expected and observed frequencies were equal 17 times, in the direction of a positive correlation 26 times, and in the direction of a negative correlation 29 times. Of the 55 cases of unequal observed and expected frequencies. the difference was only 1 in 29 cases and 2 in 15 cases. In the remaining 11 tables (7 of which showed differences of only 3), there was no apparent trend favoring any circle size. distance between forms, or $\mathrm{S}$. With no consistent trends. the small differences found between expected and observed frequencies were attributed to chance factors.

In Experiment II, only one exposure duration for each $\mathrm{S}$ was used for all eight conditions. These times were $4.7,3.2 .2 .4$, and $2.1 \mathrm{msec}$ for the four Ss. A three-way analysis of variance was performed, as in Experiment I (circle size, single-double forms, and Ss). In Experiment II, that was a slightly greater decrease in accuracy from one- to two-form displays ( $77 \%$ for one. $69 \%$ for two ) and this difference was significant $[F(1.3)$ $=10.38 . p<.05]$. The effect of circle size was also significant $[F(4.12)=4.38, p<.05]$. This was to be expected, since exposure duration was not used as a means of compensating for differential sensitivity in the fovea at various distances from center. As in Experiment I. however, these differences were rather small.

Figure 2 shows accuracy as a function of circle size and single or double forms. The drop at the $1 / 4-\mathrm{deg}$ position could be explained as some form of mutual contour inhibition due to the presence. in a relatively small area, of the letters as well as the fixation point. The $S$ effect was significant $[F(3.12)=4.1, p<.05]$. showing again some differences in overall accuracy between Ss. The interaction between circle size and single-double forms was significant $[F(4.12)=7.7$. $\mathrm{p}<.01\}$. Thus. adding another form had different effects at various distances from fixation, at least when the two forms were not equidistant from center. The Single-Double by $S$ interaction was also significant $[F(3.12)=8.2 . p<.01$, as in Experiment I, showing individual differences in ability to process two forms as compared to one. The Circle Size by $\mathrm{S}$ effect was not significant.

Given the two circle sizes, the distance between forms was determined so an analysis of variance of the two-form displays for studying interaction between conditions was not possible. Again, 2 by 2 tables for assessing correlations in sensitivity were constructed. and expected values were compared with the observed ones. As in Experiment I, the indications of correlations in sensitivity between adjacent areas were negligible. Of the 32 tables ( 8 conditions by $4 \mathrm{Ss}$ ), the observed and expected were equal three times, differed by 1 nine times, and differed by 2 nine times. Of the remaining 11 , 7 of which differed by 3 , there was no consistent trend in any condition. Where a difference occurred, 18 were in the direction of a positive correlation and 19 negative. There was no apparent tendency for even small relationships to occur more of ten with any particular $S$, foveal location, or distance between forms.

\section{DISCUSSION}

In both experiments, positive correlations in identification accuracy were expected when the forms fell on minimally separated foveal areas. The data suggest that there are two different aspects of independence to be considered. The first is whether hit rate is affected by the number of forms in a display. There was a consistent drop in accuracy from one- to two-form displays in both experiments, though nonsignificant in Experiment $I$.

One possibility for this difference is the inability of the $\mathrm{S}$ to parallel-process two forms. If the forms had to be processed sequentially, the stimulus trace or icon (Neisser, 1967) from which the forms were read would have decayed to some extent by the time the second form was read. Thus, one would expect a difference in accuracy between first and second forms reported. However, no significant differences were found between accuracy of first and second reported forms. This finding alone is not a definitive indicator of parallel vs sequential processing. but does suggest that other explanations should be explored.

Figure 1 shows that the lowest accuracy in Experiment I is found with the $1 / 4$-deg spacing. and tite next lowest with $1 / 2$ deg between the forms. Thus. it seems that some sort of mutual inhibition is taking place.

Contour masking in vision is a well-established phenomenon. Eriksen and Collins (1965) found forward. backward. and simultaneous masking of a letter stimulus by a surrounding ring. Flom. Wormouth. and Kahneman (iog.3) found that perception of the gap in al Landolt $C$ 
was interfered with by black bars placed close to the sides of the form. The larger the gap, the closer the bars had to be in order to interfere.

What is particularly interesting in the present study is that apparently the inhibition is mutual and equal between both forms in a display. One might expect a negative correlation caused by one form dominating the other. In the sense of a decrease in hit rate, a lack of independence was found for closely spaced forms. However, in the sense of momentary correlations in noise levels, independence was found for all conditions.

This does not imply that no correlations at all exist in the retina. The facts of lateral inhibition throughout the retina are well known. Howvever, the present study suggests that these effects are not strong enough or widespread enough to affect stimuli as molar as .2-deg forms. To detect the amount of correlation at the molecular level would take more sophisticated physiological apparatus rather than global behavioral measures such as form identification. The information gained in this way would be of less use for most tachistoscopic research.

These findings once again confirm the independence hypothesis for forms separated by approximately $1 \mathrm{deg}$, as found in previous studies. However, in the Collins and Eriksen (1967) study, the results for the $1 / 4$-deg condition were attributed to a positive correlation in retinal noise components. Lack of independence in this sense now seems unlikely in view of the present findings. Interaction in terms of mutual inhibition no doubt raised the exposure time for the required accuracy rate of two, three, and four forms. Apparently, the amount of inhibition was not changed in going from two- to four-form displays, and the Ss were able to parallel-process up to four forms. The different method of scoring makes it difficult to compare the two studies directly. In the present study, each form was counted individually so that if 100 double-form trials were run, the hit rate was the total number of forms correctly identified divided by 200 (the total number of forms, not displays). In the Collins and Eriksen study, all forms in a display had to be correctly identified before a trial was counted as correct. Hit rate was the proportion of correct trials, not individual forms. For example, if four forms were presented, it made no difference whether the $S$ missed one, two, three, or all four forms; the trial was counted as a single error. Some degree of differential accuracy among two-, three- and four-form displays could have been obscured by this method of counting.

Another unexpected finding in the present study was the relatively small differences in retinal sensitivity as a function of circle size. In Experiment I, there were small and nonsignificant differences in exposure duration as a function of retinal location. In Experiment II, with exposure duration held constant for all circle sizes, there was little effect on accuracy due to location for single-form stimuli (see Fig. 2). What differences in sensitivity exist. however, seem to have been magnified in the double-form displays. In Experiment II, where the two forms were not necessarily equidistant from fixation, a noticeable difference appeared among the various locations, with forms on the $1 / 2$ - and $3 / 4-\mathrm{deg}$ circles having higher hit rates. This difference is reflected in the significant Circle Size by Single-Double Form interaction in the analysis of variance. It appears that if there is any difference in clarity between the forms, the $\mathrm{S}$ will attend to the clearer one at the expense of the other, less clear form. The accuracy of both will drop from the single-form level, but the form falling on a less sensitive area will drop relatively more.

In conclusion, it seems certain that there is a mutual inhibitory effect between two close forms. This inhibition seems not to create any kind of intratrial correlation between forms. A possible follow-up study to this one might involve using the same techniques for three- and four-form displays to test for interactions and possible implications for parallel processing.

\section{REFERENCES}

Collins, J. F., \& Eriksen, C. W. The perception of multiple simultaneously presented forms as a function of foveal spacing. Perception \& Psychophysics, 1967, 2, 369-373.

Eriksen, C. W. Independence of successive inputs and uncorrelated error in visual form perception. Journal of Experimental Psychology, 1966, 72, 26-35.

Eriksen, C. W., \& Collins, J. F. Reinterpretation of one form of backward and forward masking in visual perception. Journal of Experimental Psychology, 1965, 70, 343-351.

Eriksen, C. W., \& Lappin, J. S. Independence in the perception of simultaneously presented forms at brief durations. Journal of Experimental Psychology, 1967, 73, 468-472.

Estes, W. K., \& Taylor, H. A. Visual detection in relation to display size and redundancy of critical elements. Perception \& Psychophysics, 1966, 1, 9-16.

Flom, M. C., Weymouth, F. W., \& Kahneman, D. Visual resolution and contour interaction. Journal of the Optical Society of America, 1963, 53, 1026-1032.

McNemar, Q. Psychological statistics. New York: Wiley, 1962.

Neisser, U. Cognitive psychology. New York: Appleton-Century-Crofts, 1967.

Schlosberg, H. A probability formulation of the Hunter-Sigler effect. Journal of Experimental Psychology, 1948, 38, 155-167.

(Received for publication September 18, 1972; revision received August 30, 1972.) 